\title{
Comparing an e-Health Program Vs Home Rehabilitation Program in Patients With Non- Specific Low Back Pain: Study Protocol Randomized Feasibility Trial.
}

\author{
Adelaida María Castro-Sánchez \\ Universidad de Almeria \\ Eduardo Antequera Soler \\ Universidad de Almeria \\ Guillermo A. Matarán-Peñarrocha \\ Junta de Andalucia Servicio Andaluz de Salud \\ Deirdre Hurley-Osing \\ University College Dublin \\ Manuel Fernández-Sánchez \\ Universidad de Almeria
}

Héctor García-López

Universidad de Almeria

Inmaculada C. Lara-Palomo ( $\square$ inma.lara.palomo@gmail.com )

Universidad de Almeria https://orcid.org/0000-0003-1899-2063

Study protocol

Keywords: low back pain, chronic disease, e-health, home exercise, physiotherapist opinion's

Posted Date: November 3rd, 2020

DOI: https://doi.org/10.21203/rs.3.rs-33103/v1

License: (a) (i) This work is licensed under a Creative Commons Attribution 4.0 International License.

Read Full License

Version of Record: A version of this preprint was published at Journal of Back and Musculoskeletal Rehabilitation on July 16th, 2021. See the published version at https://doi.org/10.3233/BMR-210040. 


\section{Abstract}

Background: Most patients who experience chronic low back pain are managed in primary care services, the international clinical guidelines endorse like first line of treatment the self-management and recommendations for the management of their ailment. The treatment of low back pain an individual self-maintenance program through a web application could mean maintaining of the symptoms, reducing costs in terms of medical care and time work absence. There is little evidence on the reliability of the via Internet-based rehabilitation systems to treat low back pain, the implementation of a clinical and efficient self-maintenance web program is a key priority for the health service in Andalusia.

Methods: This protocol describes a double-blind, randomized controlled feasibility trial of a telemedicine program (e-Health intervention) developed to support the self-management of people with chronic low back pain in primary care physiotherapy. Three Hospital with primary care for outpatients will be the units of randomisation, in each Hospital the participants will be randomized to one of two groups, a pragmatic control group receiving either the usual home program based on electrostimulation and McKenzie Therapy and e-Health intervention. Patients are followed up at 2 and 6 months. The primary outcomes are (1) acceptability and demand of the intervention by general practitioners, physiotherapists and patients and (2) feasibility and optimal study design/ methods for a definitive trial. Secondary outcomes will include exploratory analysis and variation in clinical outcomes of pain, disability, fear of movement, quality of life, isometric resistance of the trunk flexors, lumbar mobility in flexion and lumbar segmental range of motion; and the effect of the intervention.

Discussion: Through the specific e-Health programs at home, could increase adherence to treatment, patients could learn to control and self-manage the evolution of their low back pain, preventing its evolution to stages of greater pain and disability. If the painful symptomatology improves could be costeffective healthcare tool that can reach a large number of people living in rural or remote areas.

Trial Registration: PC-0185-2017/ NCT04283370. Registered 20 February 2020, https://clinicaltrials.gov/ct2/show/NCT04283370. Recruiting.

\section{Background}

Non-specific low back pain (LBP) is one of the most common health problems worldwide and is the leading cause of years suffering from a disability in western countries. ${ }^{1}$ Non-specific LBP is characterized by a mechanical pain of musculoskeletal origin, with no defined cause, which lasts more than 12 weeks. ${ }^{2,3}$

The most recent review in the adult general population estimated the global point prevalence at $11.9 \%$, the monthly prevalence at $23.2 \%$, and the annual prevalence at $38 \%$, being greater in females and individuals aged between 40 to 80 years. ${ }^{4,5}$ In Spain, LBP ranks first among the causes of temporary 
disability in the population over 50 years of age. ${ }^{6}$ Furthermore, as the population ages over the coming decades, it is more than likely that the number of people with non-specific LBP will increase significantly.

Although acute LBP is often reported as lasting for a relatively brief period of time and with a moderately favorable prognosis, at least $84 \%$ of the population will report symptoms of LBP at some stage in life, and around $10-20 \%$ of affected adults develop symptoms of chronic LBP (persistent pain lasting longer than three months), symptoms that persist even up to 12 months. ${ }^{7,8}$

Given its chronic nature, high rate of recurrence and equivocal management efficacy, it causes high rates of work absenteeism and visits to primary care in every industrialized society and this entails a substantial personal, community and financial burden globally. 2,6

In European countries, the total cost of LBP has been estimated at $1.7-2.1 \%$ of gross domestic product, ${ }^{6,9}$ since it is among the health problems responsible for the majority of sick leave and the top five most expensive disorders of the musculoskeletal system. ${ }^{10,11}$ In Spain, LBP accounts for over 2 million visits to a Primary Care Center (PCC) per year. ${ }^{11}$

The non-specific nature of the symptoms appears to be due to neuromusculoskeletal disorders, associated with quality of life, psychological or physical factors in relation to working conditions (hygiene conditions, ergonomic conditions and job demands). However, it is not possible to pinpoint with complete certainty the anatomical structure responsible for the symptom. ${ }^{12,13}$

European international guidelines recommend non-pharmacological treatments for chronic musculoskeletal conditions, ${ }^{14-16}$ although there is some consistency between government-sponsored guidelines and policies regarding recommendations for chronic problems, ${ }^{17}$ the health services require strong evidence of their clinical and profitability before the implementation of widespread rehabilitation programs.

Several possible interventions exist for the treatment and management of LBP, including exercise, patient education, manual therapy and electrotherapy; these are often used alone or combined. ${ }^{14,18-23}$ Transcutaneous electrical nerve stimulation (TENS) is an inexpensive nonpharmacological intervention used in the treatment of acute and chronic pain conditions. The research evidence, TENS reduces hyperalgesia through both peripheral and central mechanisms, reducing the need for medication in these patients. ${ }^{24,25}$ However, the different systematic reviews have examined the efficacy of TENS for low back pain with conflicting results. ${ }^{26-33}$ The contradiction is due often a lack of homogeneity in the studies, for example, with respect to location of TENS electrodes, frequency, intensity and duration of TENS stimulation, and the combination of TENS stimulation with another intervention. ${ }^{32}$ Deyo et al, ${ }^{33}$ examined the effectiveness of TENS, a program of stretching exercises, or a combination of both for low back pain. Although after one month patients in the exercise groups had significant improvement in self-rated pain scores and levels of activity as compared with patients in the groups that did not exercise, however, two months after the intervention when the patients interrupted the treatment, the initial improvements 
disappeared. Which suggesting that adherence to treatment is an important aspect to consider in patients with chronic pain.

International clinical practice guidelines endorse that the self-management management is the key strategy in patient care with chronic LBP, ${ }^{15,18,23}$ however, the definitions of self-management vary ${ }^{19}$. Exercises are recommended in all guidelines for chronic LBP; these determine that patients with chronic LBP should exercise and maintain a physically active lifestyle. As a result, patients typically are prescribed different types of specific back exercise (e.g. trunk coordination, endurance exercises, Mckenzie Therapy (MT), home exercise programs) and general physical activity .2,18 The specific back exercise programs have been found to be moderately effective in reducing pain and improving function in chronic LBP, especially if programs are individually designed/tailored and supervised by a physiotherapist. ${ }^{21,22}$ The MT is a treatment in which exercise is prescribed individually based on the classification made of patients with low back pain. The method classifies patients into subgroups according to their symptoms, signs and clinical examination with a series of repeated movements. The response obtained indicates which mechanical forces (movements or postures) will help reduce the problem. ${ }^{34}$ This Method of diagnosis and mechanical therapy associated with an educational component has been considered a more effective intervention in reducing pain and disability than other standard therapies in the short term (NSAIDS, educational booklet, back massage and back care advice, strength training, and spinal mobilization and general exercises). ${ }^{35-37}$ A study that compared the efficacy of Clinic-Based MT vs Telerehabilitation-Based MT in patients with chronic non-specific LBP determined that both therapies are comparable; both achieved significant effects in terms of all the treatment outcomes. ${ }^{38}$ However, the availability of secondary rehabilitation centers in the public health system could be insufficient to meet the demand of these patients in a supervised way, because of a strong correlation between the burden of musculoskeletal disease and gross domestic product per capita, with Europe being the continent where musculoskeletal diseases have the highest economic impact. ${ }^{39}$

The interventions performed electronically have been shown to be effective in subjects with chronic musculoskeletal disease, since they can provide educational information beyond traditional paper-based media, such as audio and video material that subjects can consult at any time. This facilitates goal setting, adherence, self-monitoring and behavioral and symptom-related feedback. ${ }^{40,43}$ Recent developments in mobile technology, such as mobile phone and tablet computer apps, could help in developing a platform for the delivery of self-management interventions that are adaptable, of low cost, and easily accessible. Also, the studies suggest that interventions supported by virtual materials are more accessible to patients than many traditional face-to-face services, where patient access is subject to release from family and work commitments. ${ }^{43,44}$ These data lead us to believe that patients who have the support of an online platform to perform the intervention at home, have the potential to obtain greater adherence and long-term effects than those who perform the same physiotherapy intervention without supervision in the home and without computerized support. There is also the need to explore the effectiveness, adherence, usefulness and support of interventions in primary care supported by an online platform designed exclusively for patients with chronic LBP. 


\section{Aims and objectives}

The aim of this randomized controlled trial is to evaluate the feasibility of providing an e-Health rehabilitation program through a web platform performing electroanalgesia and an exercise program following the MT for patients with chronic LBP in primary care, compared with the same home rehabilitation program but without the support of an electronic program.

Our primary objectives are: (1) To evaluate the acceptability and demand of the e-Health intervention for patients and physiotherapists in comparison with the home rehabilitation program for the optimization of their design, development and delivery; (2) to analyze the feasibility of the trial procedures, including: recruitment of patients and the most efficient and effective study design for a definitive RCT. See Table 1 below for details on feasibility aspects.

Table 1. Summary table of E-Health program content.

\begin{tabular}{|c|c|}
\hline $\begin{array}{l}\text { Session } \\
\text { number }\end{array}$ & Content \\
\hline Session 1 to 5 & $\begin{array}{l}\checkmark \text { How E- Health app works. } \\
\checkmark \text { Register: } \\
\quad \text { patients' demographics data . } \\
\text { diagnosis to be established. } \\
\checkmark \text { The app sets the objectives: description of the specific Mckenzie exercises to be } \\
\text { performed. } \\
\quad \text { Access to rationales, videos of exercise for the LBP. } \\
\text { Videos with explanation of the use of portable TENS. }\end{array}$ \\
\hline $\begin{array}{l}\text { Session } 6 \\
\text { onwards }\end{array}$ & $\begin{array}{l}\checkmark \text { Exercise program review } \\
\checkmark \text { Opportunity to modify pain changes at the beginning of each session: } \\
\cdot \quad \text { The program recalculates the recommendations and updates the treatment. }\end{array}$ \\
\hline
\end{tabular}

The secondary objectives are: (3) to assess medium-term changes in pain intensity, disability, fear of movement, quality of life, resistance of the trunk flexors, lumbar segmental range of motion in both arms.

\section{Methodology}

\section{Design and setting}


This randomized controlled feasibility trial with an allocation ratio 1:1, double-blind, clinical trial divided into two groups (Fig. 1), was designed to assess the methodology proposed for use in a definitive RCT. The protocol has been approved by the Human Research and Local Ethics Committee of the "Hospital Complex Torrecárdenas of Almeria, University Hospital Complex of Granada and Virgen Macarena de Sevilla Hospital - Andalusian Health Service" (CFS/apg). This study protocol was registered in an international clinical trial registry, clinicaltrials.gov (protocol number NCT03469024) and has followed the Standard Protocol Items: Recommendations for Interventional Trials (SPIRIT) Guidelines (Additional file 1). ${ }^{45}$

The GPs of three Hospital Centers (HC) of the Andalusian Health Service that include physical therapy in primary care for outpatients have agreed to participate. These HCs are publicly financed and include the metropolitan areas of Seville, Granada and Almeria, in Andalusia (Southern Spain).

\section{Participants}

A Consolidated Standards of Reporting Trials (or CONSORT) ${ }^{45}$ flow diagram is provided in Fig. 1. Eligible participants are intentionally selected by GPs according to the eligibility criteria provided by the research team.

\section{Inclusion criteria}

Individuals of both genders aged between 30 and 67 years old; presenting with low back pain for at least 3 months; low back pain disability $\geq 4$ on the Roland-Morris Disability Questionnaire (RMQ); and not receiving any other physiotherapy treatment are eligible for inclusion.

\section{Exclusion criteria}

Potential participants are ineligible if they have any contraindications for McKenzie exercise or electroanalgesia; presence of clinical signs or symptoms of radiculopathy; medical diagnosis of spondylolisthesis; spinal stenosis; inflammatory or metabolic disease; diagnosis of fibromyalgia; presence of central or peripheral system pathology (i.e., stroke, peripheral nerve, etc.); history of spinal surgery; or treatment with corticosteroids or other drugs in the previous 2 weeks.

\section{Identification, invitation, screening and recruitment}

This feasibility protocol is supported by the Andalusian Health Service, which is facilitating the recruitment of primary care practices for the protocol. The identification of eligible participants will be a multilevel process, established during the development phase of this project in accordance with the hospital areas of participants. The research team will provide the reference GPs with information about the trial, the inclusion and exclusion criteria of participants and the intervention groups in the study, training them to understand the importance of eligibility, and to pay attention to what is requested. After, potentially eligible patients identified by the GPs of the computerized consultation records and by chance will receive a call from a member of the research team inviting them to participate in the study. Patients who are interested in participating will receive information about the study and, if they wish, the 
investigator will provisionally evaluate their eligibility by phone. Potentially eligible patients through telephone screening will be informed of the date of the appointment with the time and place (usual hospital center) for an evaluation with the investigator. After giving their consent, potentially eligible patients will be carried out an initial examination and, if eligibility is confirmed, reference data will be collected, including self-reported outcome measures, and diagnosis according to McKenzie. Assessments face-to-face will be conducted by 2 blinded research physiotherapists (RPT) from Almeria University who have more than 10 year of experience in the clinical management of patients with chronic LBP. Within both groups, and according to the results in the face-to-face evaluation, patients will be distributed in three subgroups (postural, dysfunction and disorder) according to the therapeutic classification of McKenzie. After the evaluation, a blinded researcher will inform the participants by telephone of their assignment and will be given an appointment to begin. Participants will be invited to contact the main investigator of the project if they have any question and will be informed that they are free to withdraw from treatment and study at any stage. All participants will sign the informed consent form, prepared in compliance with the Declaration of Helsinki of the World Health Organization.

The GPs will identity patients via two methods: (1) All Patients who have consulted with LBP in the past 3 months will be identified from practice computerized records of consultations. Lists of patients identified by the search will be screened by two members of the research team who will rule out patients based on aspects of the eligibility criteria that can be determined from patient notes. (2) Patients are opportunistically identified by GPs in Hospital.

The computer software (Epidat 4.2) will automate the randomization process for this trial. The randomization sequence is automatically generated, using a table of random numbers generated by the software, which randomizes participants to each intervention group. As the software randomizes participants, a researcher will inform the participants about their assignment. The feasibility protocol will remain blind to the assignment until the full analysis is completed.

\section{Interventions}

Consenting participants will be randomly assigned in two groups to receive electroanalgesia therapy and McKenzie exercise through an E-Health program (telemedicine) or through a home rehabilitation program. Both groups will perform three sessions per week, to complete a total of 24 sessions over eight weeks. The details of the interventions are provided below.

\section{Home Rehabilitation Program}

It consists of a home rehabilitation program. Participants will receive electroanalgesia with TENS and an exercise program following the McKenzie method. At the first and second sessions, 1-hour per session, patients will be instructed by a RPT on how to use electroanalgesia and how to perform the exercises. Then, patients will be requested to perform the treatment at home 3 times per week, for 8 weeks, with a total of 24 sessions. 
Each participant will be instructed in the use of a portable TENS (TENStem eco basic, schwa-medico Medizinische Apparate Vertriebsgesellschaft mbH, Wetzlarer/35630/Ehringshausen, Deutschland) of low frequency and high phase duration $(80 \mathrm{~Hz} / 200 \mu \mathrm{s})$ applied directly in the lumbar area by means of four electrodes $(5 \times 9 \mathrm{~cm})$ at the paravertebral level bilateral. The electrostimulation treatment will be applied simultaneously to the performance of the exercises. ${ }^{33}$

The exercise program will be individualized according to the results obtained in the initial evaluation, which will determine the diagnosis and mechanical therapy of the Mckenzie Method, and which consists of the following exercises:

Patients with Postural Syndrome: exercises 1, 2 and 3 (posture correction and proxylaxis)

Patients with Dysfunction Syndrome: exercises 3, 6 and 7.

Patients with Disorder Syndrome (DS): according to the dysfunction syndrome, the following exercises are established for each subgroup:

- DS 1: exercises 1-4 and 6, with extension in recumbency.

- DS 2: the exercises will begin in the prone position and patients will continue with the DS1 protocol and exercise 5.

- DS 3: DS1 protocol, exercise 7 and rotation maintained for 2 minutes.

- DS 4: exercise 7, exercise 2 and 3, and DS 1 protocol.

- DS 5: exercise 7 and 8, and exercise 1-3.

- DS 6: DS4 Protocol, and then the protocol of DS1 and DS3.

- DS 7: exercises 7-10.

These exercises are designed to effect changes in the periarticular internal components of the spine. The exercises are described in the Fig. 2.

Each participant will be provided with support materials about the specific characteristics of the diagnosed syndrome, the intervention to be performed, with descriptive images of the Mckenzie exercises, as well as the use of TENS currents.

\section{E-Health Program}

It is a support system for the treatment of chronic LBP based on web technology, accredited as a health web. This system has a structure based on four sections: database treatment, database of user profiles, recommendations, and feedback procedures. This system allows users to register and enter a subject and modify an electroanalgesia and exercise treatment plan according to the symptomatic evolution of pain. It is based on an initial patient assessment system (Fig. 3).

Once the initial diagnosis of the patient has been made according to the clinical subtypes of low back pain according to McKenzie, the GP will be contacted to confirm that the patients are eligible to 
participate and are assigned to the correct subgroups. A multimedia database will be developed with examples of specific treatments (according to symptomatic evolution) for postural syndrome, dysfunction syndrome and derangement syndrome. The videos of the application will be shown to patients with combined electroanalgesia and exercise therapy; patients can access the platform using their computer or mobile devices with internet access. The database is configured to accommodate the application of TENS and McKenzie exercises based on the diagnosis according to the Mckenzie method, so that the treatments will be recommended by the system individually. Each clinical subgroup corresponds to a program and a series of defined exercises. Therefore, each treatment will use a VT characteristic vector with three characteristics (one for subgroup), where in each position there will be a value which indicates the degree of difficulty level for each treatment subgroup.

The system calculates and recalculates the recommendations and updates the values of the relevant recommendations for the recommended treatments. Example: the system will recommend to a subject $X$ a treatment $Y$, and according to the modification or remission of pain at the beginning of each session, it will continue with the same guideline or be modified following the therapeutic algorithm of the McKenzie method.

When the treatment is completed, an evaluation of the outcome measures will be performed and this evaluation will be registered in the system so it can subsequently make the recommendation processes.

The objective of this system, which combines the clinical characteristics of the patient with the continuous feedback of the system, is a highly accessible and specific intervention that allows the selfefficacy and behaviors and physical function of the patient to be improved.

Since many of the patients do not have a tablet to access the study platform entitled "Stop Lumbalgia", participants in the e-health group will be given a tablet to each. The patients will be requested to perform the treatment at home 3 times per week, for 8 weeks, with a total of 24 sessions.

To ensure patient adherence, control of its inputs is made to the "Stop Lumbalgia" application and the time they spend on it each login. In addition, participants of both groups are called each two weeks to remind and encourage them to perform the exercises. The Table 1 summarize the content of the program e- Health.

\section{Physiotherapist training and treatment fidelity}

The intervention will be provided by two RPTs who are experienced in trial interventions and who have 10 year of experience in the clinical management of patients with chronic LBP and academic training in McKenzie Method and electrostimulation (TENS). The RPTs will complete a 2 days of training on structure, content, evaluation and delivery of the e-Health intervention, which includes role play and comments, learning about the application and its control and a visit to the evaluation site to solve problems of the site for effective development. In addition, they will receive a set of recommendations and a list of topics that will be addressed in telephone calls made every two weeks, call time shall not exceed 10 minutes per patient. The course will be taught by a senior researcher (AMCS) who holds an 
expert in the McKenzie Method and multiple studies in chronic low back pain. Fidelity to the intervention is evaluated in both groups by recording all telephone calls and examining any systematic deviation from the protocol.

\section{Outcome measures}

At baseline, demographic data including age, gender, education, occupational and marital status and clinical presentation according a Mckenzie evaluation will be documented:

- Type of Pain: constant or intermittent, local and / or referred.

- Pain Location: Paresthesia, central, uni or bilateral symptoms.

- Interrogation: Gradual or acute onset, without apparent cause, sedentary life, previous trauma, repeated movements, history of acute crises.

- Worsens: with static load, worsening at the end of the day, during activities and movements.

- Improvement: with the change of position, in positions and during activities that do not bring the shortened tissue to the maximum range of motion.

The primary outcomes are related to the feasibility of the e- Health intervention and trial design and procedure. For participants, this will be assessed by questionnaires (see Table 2 below) of treatment acceptability and demand of treatment and trial participation. For physiotherapists, a variety of aspects of feasibility will be evaluated according to the expectations of the treatment questionnaires. 
Table 2

Feasibility aspects related to e-Health intervention and trial procedures.

Feasibility e-Health intervention Trial procedures

Acceptability Participants

The extent to which participants who have received the intervention through a web application consider that the content and support materials (web application and initial learning sessions) are appropriate and satisfactory to obtain the expected results.

Physiotherapists The extent to which the physiotherapists who have administered the intervention consider that the training, content and support materials are appropriate to meet their needs and those of their patients within the primary care service.

The extent to which participants believe that their eligibility, outcome measures, follow-up and intervention by the physiotherapist have been satisfactory.

physiotherapists who have
administered the intervention
consider that the training, content
appropport materials are
and those of their patients within
the primary care service.

General

Practitioners

The extent to which the GPs who have carried out the first screening of patients, consider that the eligibility criterio and recruitment are suitable for detecting the potential sample.

$\begin{array}{ll}\text { Demand Participants } & \begin{array}{l}\text { The extent to which participants } \\ \text { adhere to the e-Health intervention, } \\ \text { complying with the weekly } \\ \text { sessions. }\end{array}\end{array}$

Physiotherapists The extent to which physiotherapists perceive the demand to complete their tasks required to participate in the trial, including intervention procedures.

Practicality Physiotherapists

The extent to which participants perceive the burden of participating in follow-up and completing specific outcome measures within the trial.

The extent to which physiotherapists perceive the demand of completing their required tasks for participating in the trial.

The factors that influence the implementation of the e-health intervention in a variety of health environments due to variations in personnel, facilities, equipment and the environment. 
Feasibility

Adaptation Participants

The extent to which the content of the e-health intervention, support materials and learning classes should be modified to improve their acceptability and implementation for a future definitive trial

\section{Trial procedures}

The extent to which recruitment, follow-up procedures and the number and outcome measures should be modified during / at the end of the trial to improve its acceptability and implementation for a definitive future trial.

Physiotherapists

The extent to which the e-health intervention training, the content of the program, the classes previously conducted for its learning and the support materials (web application) should be modified during / at the end of the test to improve its acceptability and implementation for a definitive future proof.
The extent to which the recruitment and fidelity procedures of the trial, including the tasks of physiotherapists, should be modified during / at the end of the trial to improve the acceptability and implementation of a definitive future trial.

In addition, the feasibility of the design and trial procedures will be assessed through experimentation of methodological procedures, recruitment, intervention procedures, the number and reasons for withdrawal during the treatment process, the feasibility outcome measurement, follow-up and fidelity procedures, refining factors that influence the implementation of the intervention.

The selected secondary outcome measures are the disability will be evaluated using Roland Morris Disability Questionnaire (RMDQ) ${ }^{46,47}$ and the Oswestry Disability Index (ODI); ${ }^{48,49}$ quality of life will be assessed using the SF-36 Quality of Life Questionnaire ${ }^{50,51}$ and the EuroQol (EQ) 5D-5L; ${ }^{52,53}$ the pain intensity and days that pain incapacitates for work by the by Visual Analogic Scale (VAS) ${ }^{54,55}$ and an ítem developed specifically for this feasibility protocol; kinesiophobia (fear of movement and re-injury) will be assessed using the Tampa Scale Kinesiophobia (TSK), ${ }^{56,57}$ isometric resistance of the abdominal muscles will evaluated by the McQuade test, ${ }^{58}$ and range of motion of the trunk in flexion and in the sagittal plane by the fingertip-to-floor test and the SpinalMouse ${ }^{\circledR} .{ }^{59,60}$

Measurement variables will be evaluated before the first treatment session (baseline data), after the 8week intervention period (immediately after the last session, i.e. 2 months), and after six months after the last session (follow-up). See Table 3 for full details of measures included. 
Table 3

Variables, measures and their characteristics.

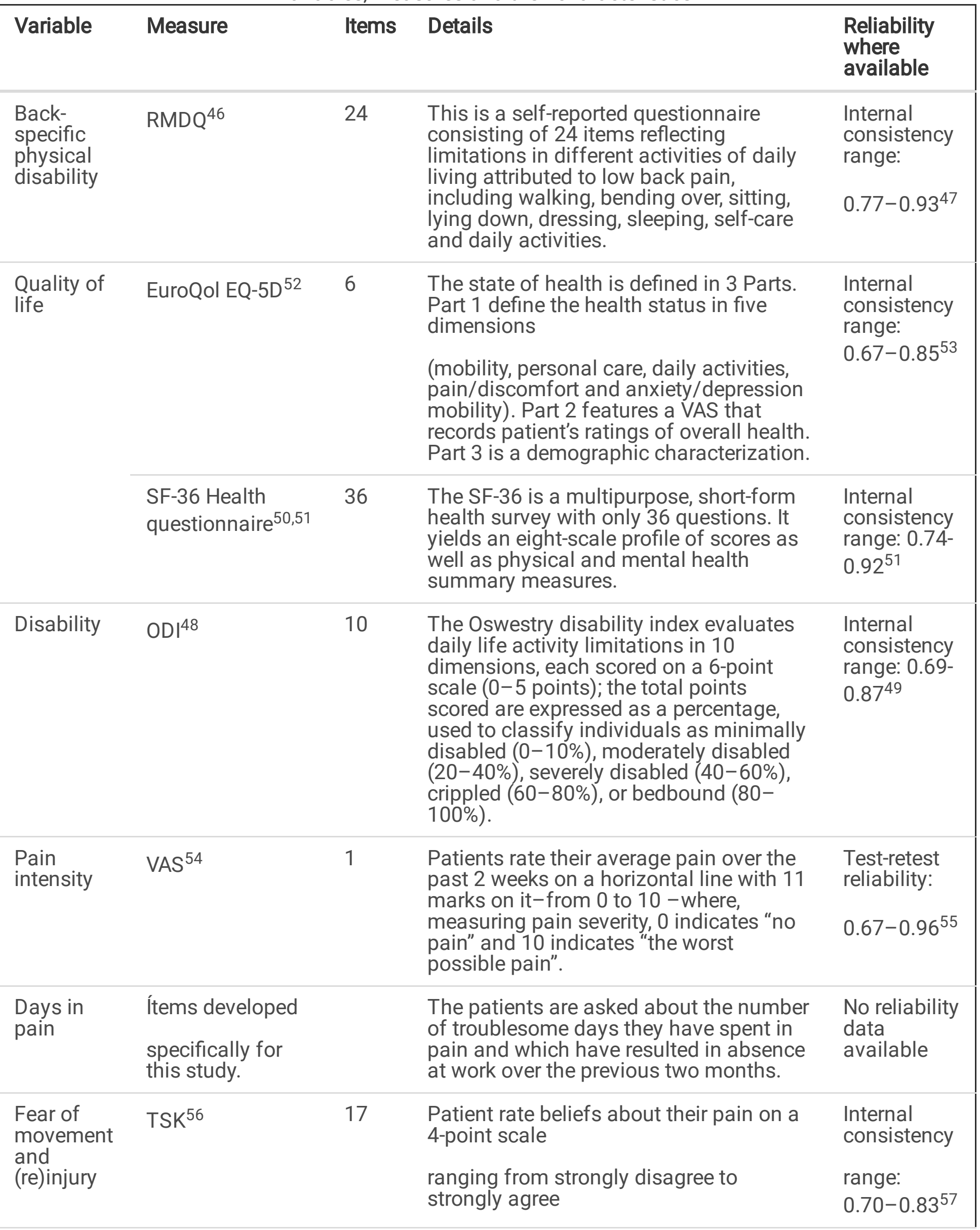




\begin{tabular}{|c|c|c|c|c|}
\hline Variable & Measure & Items & Details & $\begin{array}{l}\text { Reliability } \\
\text { where } \\
\text { available }\end{array}$ \\
\hline $\begin{array}{l}\text { Isometric } \\
\text { resistance } \\
\text { of } \\
\text { abdominal } \\
\text { muscles }\end{array}$ & McQuade Test ${ }^{58}$ & 1 & $\begin{array}{l}\text { The purpose of this test is to compile the } \\
\text { times of isometric resistance of the } \\
\text { subjects by performing a trunk flexion } \\
\text { exercise with flexed knees, and the } \\
\text { patient supine. }\end{array}$ & $\begin{array}{l}\text { Internal } \\
\text { consistency: } \\
0.97^{58}\end{array}$ \\
\hline $\begin{array}{l}\text { Forward } \\
\text { bending }\end{array}$ & $\begin{array}{l}\text { Fingers-floor } \\
\text { distance } 59\end{array}$ & 1 & $\begin{array}{l}\text { The patient flexes the trunk forward from } \\
\text { the standing position, and the distance } \\
\text { from the fingers to the ground is } \\
\text { measured. }\end{array}$ & $\begin{array}{l}\text { Internal } \\
\text { consistency: } \\
0.82^{59}\end{array}$ \\
\hline $\begin{array}{l}\text { Lumbar } \\
\text { mobility }\end{array}$ & $\begin{array}{l}\text { Range of motion } \\
\text { and lumbar } \\
\text { segmental } \\
\text { mobility } 60\end{array}$ & & $\begin{array}{l}\text { This variable is quantified using the } \\
\text { SpinalMouse } \AA \text { device (Phisiotech, } \\
\text { Spain). It is an electronic computer-aided } \\
\text { measuring device that measures sagittal } \\
\text { spinal amplitude of movement (ROM) } \\
\text { and intersegmental angles in a non- } \\
\text { invasive way. }\end{array}$ & $\begin{array}{l}\text { Internal } \\
\text { consistency } \\
\text { range: } \\
0.92-0.95^{60}\end{array}$ \\
\hline $\begin{array}{l}\text { Adherence } \\
\text { to specific } \\
\text { activities } \\
\text { for LBP }\end{array}$ & $\begin{array}{l}\text { Brief } \\
\text { questionnaire } \\
\text { developed for this } \\
\text { trial. }\end{array}$ & 6 & $\begin{array}{l}\text { Patients are asked about the number of } \\
\text { weeks that they have fully completed the } \\
\text { program. They are also asked for an } \\
\text { estimate of how many days a week they } \\
\text { did Mckenzie exercises and the } \\
\text { electroanalgesia protocol with TENS. } \\
\text { Patients are also asked if they stopped } \\
\text { doing the activities because they are no } \\
\text { longer experiencing pain. }\end{array}$ & $\begin{array}{l}\text { No reliability } \\
\text { data } \\
\text { available }\end{array}$ \\
\hline $\begin{array}{l}\text { *Abbrevia } \\
\text { Disability } \\
\text { Scale for }\end{array}$ & Q-5D-3L: Euro & In & $\begin{array}{l}\text { ns } 3 \text { Levels; RMDQ = Roland-Morris Lov } \\
\text { ility Index; VAS = Visual Analogue Scale } \\
\text { xperiences of Therapy Scale. }\end{array}$ & $\begin{array}{l}\mathrm{k} \text { and } \\
=\text { Tampa }\end{array}$ \\
\hline
\end{tabular}

The patient's adherence to the internet intervention will be explored by a brief questionnaire developed for this trial that will examine the data of use of the objective intervention automatically collected by the internet intervention E-Health. These data provide specific information on the number of logins, the number of sessions accessed, the objectives of the established treatment, the exercise module accessed and the time spent on each web page. While the group that performs the program at home without electronic support will use a diary to record the same data as the E-Health group.

\section{Timeline}

The recruitment of patients started on 15 February 2020 and was completed by 30 June 2020. All data for all follow-up occasions is expected to be collected before to 30 October 2020 . The data analysis, writing of scientific manuscripts and submissions to peer-reviewed scientific journals will be carried out during 2020 and 2021. A summary of the study outline is given in Table 4. 
Table 4

Schedule of enrolment, interventions and assessments.

TIMEPOINT
STUDY PERIOD

Enrolment Allocation Post-allocation

Pre first
visit

$\begin{array}{llll}\begin{array}{l}\text { Pre first } \\ \text { visit and } \\ \text { assessment }\end{array} & \text { Baseline } & 2 \text { months } & 6 \\ & & \begin{array}{l}\text { (immediate } \\ \text { post- } \\ \text { intervention) }\end{array} & \\ & & \end{array}$

\section{ENROLMENT:}

Eligibility screen GPs $x$

Eligibility screen by phone RPTs $\quad X$

Eligibility screen face to face $\mathrm{X}$

Informed consent $\mathrm{X}$

Allocation

$X$

INTERVENTIONS:

Home program

E- health program (telemedicine)

ASSESSMENTS:

Age, gender, education, occupational and marital status.

Clinical presentation of pain according McKenzie (type, location, interrogation, worsens, improvement)

Feasibility of the e-Health intervention and trial design and procedure

RMDQ, ODI, SF-36, EQ-5D, VAS, TSK, McQuade Test, Fingertip-tofloor and SpinalMouse.

$\begin{array}{lll}x & x & x \\ x & x & x\end{array}$

$\mathrm{X}$

$X$

$\mathrm{X} \quad \mathrm{X}$

X $\quad x \quad x$

\section{Sample size}

The sample size was calculated according to the specifications established by Willian. ${ }^{61}$ Assuming a standard deviation of 2.5 points, a 2-tailed test, an alpha (a) level of 0.05 , and a desired potency (beta) of $85 \%$, the estimated sample size was 270 patients with low back pain per group to detect a 2.5 point absolute difference in the primary outcome measure the in the RMQ (MCID) (estimated for a variance in patients with chronic LBP of 10 points), ${ }^{62}$ score at 6 months post intervention between the group 
intervention and home rehabilitation arms. In each study center (Almería, Granada and Sevilla) 180 patients with chronic low back pain will be recruited. There will be a minimum of three groups in each arm, participating in two waves of recruitment with the objective of recruiting forty-five participants in each group per wave, resulting in 540 participants with chronic low back pain ( 90 per arm).

Recommendations for feasibility studies suggest that at least 30 participants per arm be included for the analysis of the data set. ${ }^{63-65}$ If the effect of the cluster design is taken into account and an ICC coefficient of 0.03 is assumed, an effective sample size of 30 would require 36 participants per arm. ${ }^{66}$ Given a $25 \%$ loss during follow-up, we would need to recruit 48 participants per arm (96 in total). Therefore, this study size will be large enough to allow an accurate estimate of the ICC coefficient.

\section{Data analysis plan}

An a priori data analysis plan will be implemented by the trial statistician on completion of data collection. The test statistician will carry out an a priori data analysis plan at the end of the data collection. For the primary analysis, as it is a feasibility study, an exhaustive descriptive analysis of the data is performed. A combination of quantitative and qualitative methods will be used to respond to the objectives related to the feasibility of the intervention and the trial procedures both from the point of view of the participants and the RPTs. These data will determine the feasibility and will improve the study design for a future definitive trial.

Data will be analyzed with SPSS@b version 21.0 and STATA 14, it will follow intention-to-treat principles. The analysis of the data of the secondary outcome measure will be carried out at the end of the trial and will be performed by the statistician who will remain blinded to the identification of the group until the analysis is completed. Baseline demographic and clinical variables will be examined between both groups' independent Student t-test for continuous data and $\chi 2$ tests of independence for categorical data in the parametric variables, and through the Mann-Whitney $U$ for non-parametric data. The data normality will be tested through the Kolmogorov-Smirnov test and if we find non-normally distributed data, we will use the Kruskal-Wallis test. To investigate the effect of treatment (E-Health vs Home rehabilitation) and the interaction terms between treatment group versus time, the repeated measures analysis of variance (ANOVA) with time (baseline, at 2 (post-treatment) and 6 months follow up (after last session) will be used. The confidence interval will be established at $95 \%$, and the significance level at 0.05 .

\section{Adverse effects}

The risk of adverse events occurring as a consequence of the interventions in this trial is low. All activities and their intensity (specific McKenzie exercises for the lower back and TENS) will be recommended based on the individual signs and symptoms of each participant and will be described in detail in paper format or through internet support in group e -health. Patients will be reminded that the assigned level should be comfortable for them. It will be amended quickly if the patient feels that the initial level is too high. 
Participants must inform the RPTs of any adverse effects / events, and they will be responsible for communicating it immediately to the IP. The treatment will be modified or interrupted if necessary, and the type, frequency and duration of the effect will be documented if it occurs.

\section{Ethics, data security and dissemination}

All patients, GPs and RPTs will receive specific information on the study in writing and will have a chance to discuss procedures with a member of the study team before consenting to take part. They will also be informed that they can leave the study at any point. Participants that agree to participate in the study will sign two copies of the informed consent, one that will be kept in the trial records and one for the participant. Informed consent has been obtained for the clinical and personal images and details of patients included in this study (Figs. 2 and 3).

The data collected from each patient will be stored in a closed locker in an office of the University of Almeria and only the RPTs evaluators will have access to that information. Subsequently, the data will be entered and saved by the statistician on a laptop with password protection to maintain confidentiality. Eligibility criteria, results and analysis will not be modified after registration of the first participant.

The feasibility results will be published in journals indexed in Journal citations report and presented at national and international conferences.

\section{Discussion}

In this randomized controlled trial we intend investigate the effectiveness of an e-Health programs versus home rehabilitation programs in patients with chronic LBP. The difference between both programs is that the e-Health group has constant and remote information on the exercises through a web platform.

Considering that the adherence to home exercise programmes ranges from $50-70 \% 67,68$, and that some studies have shown that patients who do not adhere to home exercise regimens benefit less from treatment than those that do ${ }^{69}$. That lack of adherence to treatment in patients with low back pain could be facilitated by using computer systems to make exercise programs more attractive ${ }^{70}$. Previous studies show that patients prefer short, simple exercise programmes, and prefer their therapist to be knowledgeable about their disease, encourage feedback, motivate them to learn, give them reminders and monitor their results and adherence to the programme ${ }^{71}$. As can be seen in Table 2 , also through this study we intend to know the preferences and adherence of the patient and the opinion of physiotherapists and GPs on the e-Health intervention (web applications and learning sessions), maintaining a constant feedback with the patient, and recording if the sessions are appropriate and satisfactory to obtain the expected results.

If data are obtained on patient preferences, and adequate feedback is given to achieve adherence, the new technologies could allow physical therapists to provide their patients with the treatment, follow-up and remote contact they require. 
Through the specific e-Health programs at home, could increase adherence to treatment, patients could learn to control and self-manage the evolution of their LBP, preventing its evolution to stages of greater pain and disability. If the painful symptomatology improves could be cost-effective healthcare tool that can reach a large number of people living in rural or remote areas.

\section{Possible limitations of the study}

The main limitation of the present study is the problem of adherence to the E-Health program due to the difficulty of accessing the web application in certain population centers, such as those belonging to rural population groups. This limitation is mitigated by providing 15 tablets of 10,1" Quad Core (Supernova Qi16, Leotec) in each study center, together with a 5-session course on their use.

\section{Trial status}

Protocol version number ID PC-0185-2017/ NCT04283370 and date February 20, 2020. The recruitment started February 25, 2020 and will be completed July 30, 2020.

\section{Abbreviations}

CONSORT: Consolidated Standards of Reporting Trials; DS: Disorder Syndrome; LBP: low back pain; ODI: Oswestry Disability Index; RPT: Research Physiotherapists; RMDQ: Roland-Morris Low Back and Disability Questionnaire; SF-36: short form health survey questionnaire; SPSS: Software statistical package for the social sciences; TENS: transcutaneous electrical nerve stimulation; TSK: Tampa scale of kinesiophobia; VAS = Visual Analogue Scale.

\section{Declarations}

Competing interests. The authors declare that they have no competing interests.

Authors' contributions. AMCS: Conceptualization, methodology, writing-original draft, writing-review and editing, supervision, and project administration. ICLP: Conceptualization, methodology, investigation, formal analysis, and writing-review and editing. EAS, GAMP, DHO, MFS and HGL: Conceptualization, methodology, and writing-review and editing. All authors read and approved the final manuscript.

Patient consent for publication. Not required.

Ethics approval. All participants provided written informed consent to participate in the study. Ethical approval was obtained from Human Research and Local Ethics Committee of the "Andalusian Health Service", PC-0185-2017.

Acknowledgements. Not applicable.

Funding. This work was supported by a research project grant (PI18/00562 Proyecto E-CEPEDOL) from the Carlos III Health Institute and two grant from the Andalusian Health Service, Junta de Andalucía (PC- 
0185-2017 and PC-0536-2017). But these have not been part in designing the study, in its implementation, analysis, data interpretation and presentation of the results.

\section{Availability of data and materials. Not applicable.}

Provence and peer review. Not commissioned, peer reviewed for ethical and funding approval prior to submission.

\section{References}

1. Krismer M, van Tulder M. Low back pain (non-specific). Best Pract Res Clin Rheumatol. 2007; 21:7791.

2. Delitto A, George SZ, Van-Dillen L, Whitman JM, Sowa GA. Low back pain: Clinical practice guidelines linked to the international classification of functioning, disability, and healh for the Orthopaedic section of the American Physical Therapy Association. J Orthop Phys Ther. 2012; 42(4): A1-57.

3. Wolter T, Szabo E, Becker R, Mohadjer M, Knoeller SM. Chronic low back pain: course of disease from the patient's perspective. Int Orthop. 2011; 35:717-724.

4. Mamchikanti L, Singh V, Falco F, Benyamin R, Hirsch J. Epidemiology of low back pain in adults. 2014; 17: 3-10.

5. Hoy D, Bain C, Williams G, March L, Brooks P, Blyth F, Woolf A, Vos T, Buchbinder R. A systematic review of the global prevalence of low back pain. Arthritis Rheum. 2012 Jun;64(6):2028-37. doi: 10.1002/art.34347. Epub 2012 Jan 9. Review. PubMed PMID: 22231424.

6. Walker B. The prevalence of low back pain: a systematic review of the literature from 1966 to 1998 . J Spinal Disord 2000;13:205-17. Texto Completo OVID Catálogo Almirez Enlaces Bibliograficos [Context Link]

7. Costa LCM, Maher CG, Hancock MJ, et al. The prognosis of acute and persistent low-back pain: a meta-analysis. CMAJ 2012;184: E613-24.

8. Costa LCM, Maher CG, McAuley JH, Hancock MJ, Herbert RD, Refshauge KM, Henschke N. Prognosis for patients with chronic low back pain: inception cohort study. 2009;339:b3829.

9. Deyo R, Cherkin D, Conrad D, et al. Cost, controversy, crisis: low back pain and the health of the public. Annu Rev Public Health 1991;12:141-56.

10. Van Tulder M, Koes B, Bombardier C. Low back pain. Best Pract Res Clin Rheumatol 2002;16:761-75.

11. Kovacs F, Fernandez C, Cordero A, et al; Spanish Back Pain Research Network. Non-specific low back pain in primary care in the Spanish National Health Service: a prospective study on clinical outcomes and determinants of management. BMC Health Serv Res 2006;6:57.

12. Yang H, Haldeman S, Lu ML, Baker D. Low back pain prevalence and related workplace psychosocial risk factors: a study using data from the 2010 National Health Interview Survey. J Manipulative Physiol Ther. 2016; 39 (7): 459-472. 
13. Waddell G, Burton AK. Occupational health guidelines for the management of low back pain at work: evidence review. Occup Med. 2001; 51: 124-135.

14. Almeida M, Saragiotto B, Maher CG. Primary care management of non-specific low back pain: key messages from recent clinical guidelines. Med J Aust. 2018 Sep;209(5):235-235.e1.

15. Chenot JF, Greitemann B, Kladny B, Petzke F, Pfingsten M, Schorr SG. Non-Specific Low Back Pain. Dtsch Arztebl Int. 2017 Dec 25;114(51-52):883-890.

16. Herman PM, Lavelle TA, Sorbero ME, Hurwitz EL, Coulter ID. Are Nonpharmacologic Interventions for Chronic Low Back Pain More Cost Effective Than Usual Care? Proof of Concept Results From a Markov Model. Spine (Phila Pa 1976). 2019 Oct 15;44(20):1456-1464. doi:

10.1097/BRS.0000000000003097. PubMed PMID: 31095119; PubMed Central PMCID: PMC6779140.

17. Cheng JS, Lee MJ, Massicotte E, Ashman B, Gruenberg M, Pilcher LE, Skelly AC. Clinical guidelines and payer policies on fusion for the treatment of chronic low back pain. Spine (Phila Pa 1976). 2011 Oct 1;36(21 Suppl):S144-63.

18. Traeger A, Buchbinder R, Harris I, Maher $C$. Diagnosis and management of low-back pain in primary care. CMAJ. 2017;189(45):E1386-E1395. doi:10.1503/cmaj.170527.

19. May S. Self-management of chronic low back pain and osteoarthritis. Nat Rev Rheumatol 2010;6:199-209.

20. National Institute for Health and Clinical Excellence. Low back pain and sciatica in o ow back pain and sciatica in over 16s: assessment and management assessment and management. NICE guideline Published: 30 November 2016 Available at: www.nice.org.uk/guidance/ng59

21. Hayden JA, van Tulder MW, Malmivaara AV, Koes BW. Meta-Analysis: exercise therapy for nonspecific low back pain. Ann Intern Med 2005;142:765-75.

22. van Middelkoop M, Rubinstein SM, Kuijpers $T$, et al. A systematic review on the effectiveness of physical and rehabilitation interventions for chronic non-specific low back pain. Eur Spine $J$ 2011;20:19-39.

23. Delitto A, George SZ, Van Dillen L, Whitman JM, Sowa G, Shekelle P, Denninger TR, Godges JJ. Low back pain. J Orthop Sports Phys Ther. 2012 Apr;42(4):A1-57. doi: 10.2519/jospt.2012.42.4.A1. Epub 2012 Mar 30. PubMed PMID: 22466247; PubMed Central PMCID: PMC4893951.

24. Köke AJ, Schouten JS, Lamerichs-Geelen MJ, Lipsch JS, Waltje EM, van Kleef M, Patijn J. Pain reducing effect of three types of transcutaneous electrical nerve stimulation in patients with chronic pain: a randomized crossover trial. Pain. 2004 Mar;108(1-2):36-42.

25. Jauregui JJ, Cherian JJ, Gwam CU, Chughtai M, Mistry JB, Elmallah RK, Harwin SF, Bhave A, Mont MA. A Meta-Analysis of Transcutaneous Electrical Nerve Stimulation for Chronic Low Back Pain. Surg Technol Int. 2016 Apr;28:296-302. Review. PubMed PMID: 27042787.

26. Dailey DL, Rakel BA, Vance CG, et al. Transcutaneous electrical nerve stimulation reduces pain, fatigue and hyperalgesia while restoring central inhibition in primary fibromyalgia. Pain. 2013;154:2554-2562. 
27. DeSantana JM, da Silva LF, De Resende MA, Sluka KA. Transcutaneous electrical nerve stimulation at both high and low frequencies activates ventrolateral periaqueductal grey to decrease mechanical hyperalgesia in arthritic rats. Neuroscience. 2009;163:1233-1241

28. Walsh DM, Howe TE, Johnson MI, Sluka KA. Transcutaneous electrical nerve stimulation for acute pain. Cochrane Database Syst. Rev. 2009;2:CD006142. [PubMed] [Google Scholar]

29. Khadilkar A, Odebiyi DO, Brosseau L, Wells GA. Transcutaneous electrical nerve stimulation (TENS) versus placebo for chronic low-back pain. Cochrane Database Syst. Rev. 2008;4:CD003008. [PubMed] [Google Scholar]

30. Machado LA, Kamper SJ, Herbert RD, Maher CG, McAuley JH. Analgesic effects of treatments for non-specific low back pain: a meta-analysis of placebo-controlled randomized trials. Rheumatology (Oxford) 2009;48:520-527. [PubMed] [Google Scholar]

31. Claydon LS, Chesterton LS. Does transcutaneous electrical stimuation (TENS) produce 'doseresponse'? A review of systematic reviews on chronic pain. Phys. Ther. Rev. 2008;13(6):450-463. [Google Scholar] - A systematic review of TENS trials in multiple chronic pain conditions with attention to TENS dose, protocol differences, and statistical power.

32. Vance CG, Dailey DL, Rakel BA, Sluka KA. Using TENS for pain control: the state of the evidence. Pain Manag. 2014;4(3):197-209. doi:10.2217/pmt.14.13

33. Deyo RA, Walsh NE, Martin DC, Schoenfeld LS, Ramamurthy S. A controlled trial of transcutaneous electrical nerve stimulation (TENS) and exercise for chronic low back pain. N. Engl. J. Med. 1990;322:1627-1634.

34. Mann SJ, Singh P. McKenzie Back Exercises. 2019 Jan;. Review. PubMed PMID: 30969542.

35. Clare HA, Adams R, Maher CG. A systematic review of efficacy of McKenzie therapy for spinal pain. Aust J Physiother. 2004;50(4):209-16. Review. PubMed PMID: 15574109).

36. Busanich BM, Verscheure SD. Does McKenzie therapy improve outcomes for back pain?. J Athl Train. 2006 Jan-Mar;41(1):117-9.

37. Garcia AN, Costa Lda C, da Silva TM, Gondo FL, Cyrillo FN, Costa RA, Costa LO. Effectiveness of back school versus McKenzie exercises in patients with chronic nonspecific low back pain: a randomized controlled trial. Phys Ther. 2013 Jun;93(6):729-47.

38. Mbada CE, Olaoye MI, Dada OO, Ayanniyi O, Johnson OE, Odole AC, Ishaya GP, Omole OJ, Makinde MO. Comparative Efficacy of Clinic-Based and Telerehabilitation Application of Mckenzie Therapy in Chronic Low-Back Pain. Int J Telerehabil. 2019 Spring;11(1):41-58.

39. Sebbag E, Felten R, Sagez F, Sibilia J, Devilliers H, Arnaud L. The world-wide burden of musculoskeletal diseases: a systematic analysis of the World Health Organization Burden of Diseases Database. Ann Rheum Dis. 2019 Jun;78(6):844-848.

40. Cottrell MA, O'Leary SP, Raymer M, Hill AJ, Comans T, Russell TG. Does telerehabilitation result in inferior clinical outcomes compared with in-person care for the management of chronic musculoskeletal spinal conditions in the tertiary hospital setting? A non-randomised pilot clinical trial. J Telemed Telecare. 2019 Nov 26;:1357633X19887265. 
41. Hurley MV, Walsh N, Bhavnani V, Britten N, Stevenson F. Health beliefs before and after participation on an exercised-based rehabilitation programme for chronic knee pain: doing is believing. BMC Musculoskelet Disord. 2010;11:31.

42. Palacín-Marín F, Esteban-Moreno B, Olea N, Herrera-Viedma E, Arroyo-Morales M. Agreement between telerehabilitation and face-to-face clinical outcome assessments for low back pain in primary care. Spine (Phila Pa 1976). 2013 May 15;38(11):947-52.

43. Geraghty AWA, Stanford R, Stuart B, Little P, Roberts LC, Foster NE, Hill JC, Hay EM, Turner D, Malakan W, Leigh L, Yardley L. Using an internet intervention to support self-management of low back pain in primary care: findings from a randomised controlled feasibility trial (SupportBack). BMJ Open. 2018 Mar 9;8(3):e016768.

44. Lindberg B, Nilsson C, Zotterman D, Söderberg S, Skär L. Using Information and Communication Technology in Home Care for Communication between Patients, Family Members, and Healthcare Professionals: A Systematic Review. Int J Telemed Appl. 2013;2013:461829.

45. Chan A-W, Tetzlaff JM, Altman DG, Laupacis A, Gøtzsche PC, Krleža-Jerić K, Hróbjartsson A, Mann H, Dickersin K, Berlin J, Doré C, Parulekar W, Summerskill W, Groves T, Schulz K, Sox H, Rockhold FW, Rennie D, Moher D. SPIRIT 2013 Statement: Defining standard protocol items for clinical trials. Ann Intern Med. 2013;158(3):200-207

46. Roland M, Fairbank J. The Roland-Morris Disability Questionnaire and the Oswestry Disability Questionnaire. Spine (Phila Pa 1976) 2000;25:3115-24.

47. Muller U, Duetz MS, Roeder C, et al. Condition-specific outcome measures for low back pain. Part I: validation. Eur Spine J 2004;13:301-13.

48. Ferrer M, Pellisé F, Escudero O, Alvarez L, Pont A, Alonso J, Deyo R. Validation of a minimum outcome core set in the evaluation of patients with back pain. Spine (Phila Pa 1976). 2006 May 20;31(12):1372-9.

49. Chiarotto A, Maxwell LJ, Terwee CB, Wells GA, Tugwell P, Ostelo RW. Roland-Morris Disability Questionnaire and Oswestry Disability Index: Which Has Better Measurement Properties for Measuring Physical Functioning in Nonspecific Low Back Pain? Systematic Review and MetaAnalysis. Phys Ther. 2016 Oct;96(10):1620-1637.

50. Ware JE. SF-36 health survey update. Spine (Phila Pa 1976). 2000; 25(24):3130-3139.

51. Alonso J, Prieto L, Antó JM. [The Spanish version of the SF-36 Health Survey (the SF-36 health questionnaire): an instrument for measuring clinical results]. Med Clin (Barc). 1995; 104(20):771-776.

52. Rabin R, Charro F. EQ-SD: a measure of health status from the EuroQol Group. Ann Med. 2009;33(5):337-4.

53. Ye Z, Sun L, Wang Q. A head-to-head comparison of EQ-5D-5 L and SF-6D in Chinese patients with low back pain. Health Qual Life Outcomes. 2019 Apr 11;17(1):57. doi: 10.1186/s12955-019-1137-6.

54. Crichton N. Visual analogue scale (VAS). J Clin Nurs. 2001; 10(5): 706-706.

55. Kahl C, Cleland JA. Visual analogue scale, numeric pain rating scale, and the McGill pain questionnaire: an overview of psychometric properties. Phys Ther Rev 2005;10:123-8. 
56. Kori SH, Miller RP, Todd DD. Kinesiophobia: a new view of chronic pain behavior. Pain Manag 1990;3:35-43.

57. Swinkels-Meewisse EJ, Swinkels RA, Verbeek AL, et al. Psychometric properties of the Tampa Scale for kinesiophobia and the fear-avoidance beliefs questionnaire in acute low back pain. Man Ther 2003;8:29-36.

58. McGuill SM, Childs A, Liebenson C, Endurace times for low back stabilization exercises: clinical targets for testing and training from a normal database. Arch Phys Med Rehabil. 1999; 80: 941-944.

59. Frost M, Sutckey S, Samelley LA. Dorman G. Reliability of measuring trunk motions in centimetres. Phys Ther. 1982; 62: 1431-1438.

60. Post RB, Leferink VJM. Spinal mobility: sagittal range of motion measured with the SpinalMouse, a new non-invasive device. Archives of Orthopaedic and Trauma Surgery. 2004;124(3):187-192.

61. Willan AR. Analysis, sample size, and power for estimating incremental net health benefit from clinical trial data. Control Clin Trials. 2001 Jun;22(3):228-37.

62. Ostelo RW, de Vet HC (2005) Clinically important outcomes in low back pain. Pract. Res. Clin Rheumatol 19: 593-607.

63. Browne RH. On the use of a pilot sample for sample-size determination. Stat Med 1995;14:1933-40.

64. Lancaster GA, Dodd S, Williamson PR. Design and analysis of pilot studies: recommendations for good practice. J Eval Clin Pract 2004;10:307-12.

65. Shih WJ, Ohman-Strickland PA, Lin Y. Analysis of pilot and early phase studies with small sample sizes. Stat Med 2004;23:1827-42.

66. Lonsdale C, Hall AM, Williams GC, et al. Communication style and exercise compliance in physiotherapy (CONNECT). A cluster randomized controlled trial to test a theory-based intervention to increase chronic low back pain patients' adherence to physiotherapists' recommendations: study rationale, design, and methods. BMC Musculoskelet Disord 2012;13:104.

67. Holden MA, Haywood KL, Potia TA, Gee M, McLean S. Recommendations for exercise adherence measures in musculoskeletal settings: a systematic review and consensus meeting (protocol). Syst Rev. 2014;3:10.

68. Medina-Mirapeix F, Escolar-Reina P, Gascon-Canovas JJ, Montilla-Herrador J, Jimeno-Serrano FJ, Collins SM. Predictive factors of adherence to frequency and duration components in home exercise programs for neck and low back pain: an observational study. BMC Musculoskelet Disord. 2009;10:155.

69. Thomas KS, Muir KR, Doherty M, Jones AC, O'Reilly SC, Bassey EJ. Home based exercise programme for knee pain and knee osteoarthritis: randomised controlled trial. 2002;325(7367):752.

70. Palazzo C, Klinger E, Dorner V, Kadri A, Thierry O, Boumenir Y, et al. Barriers to home-based exercise program adherence with chronic low back pain: Patient expectations regarding new technologies. Ann Phys Rehabil Med. 2016;59(2):107-13. 
71. Escolar-Reina P, Medina-Mirapeix F, Gascon-Canovas JJ, Montilla-Herrador J, Jimeno-Serrano FJ, de Oliveira Sousa SL, et al. How do care-provider and home exercise program characteristics affect patient adherence in chronic neck and back pain: a qualitative study. BMC Health Serv Res.

2010;10:60.

\section{Figures}

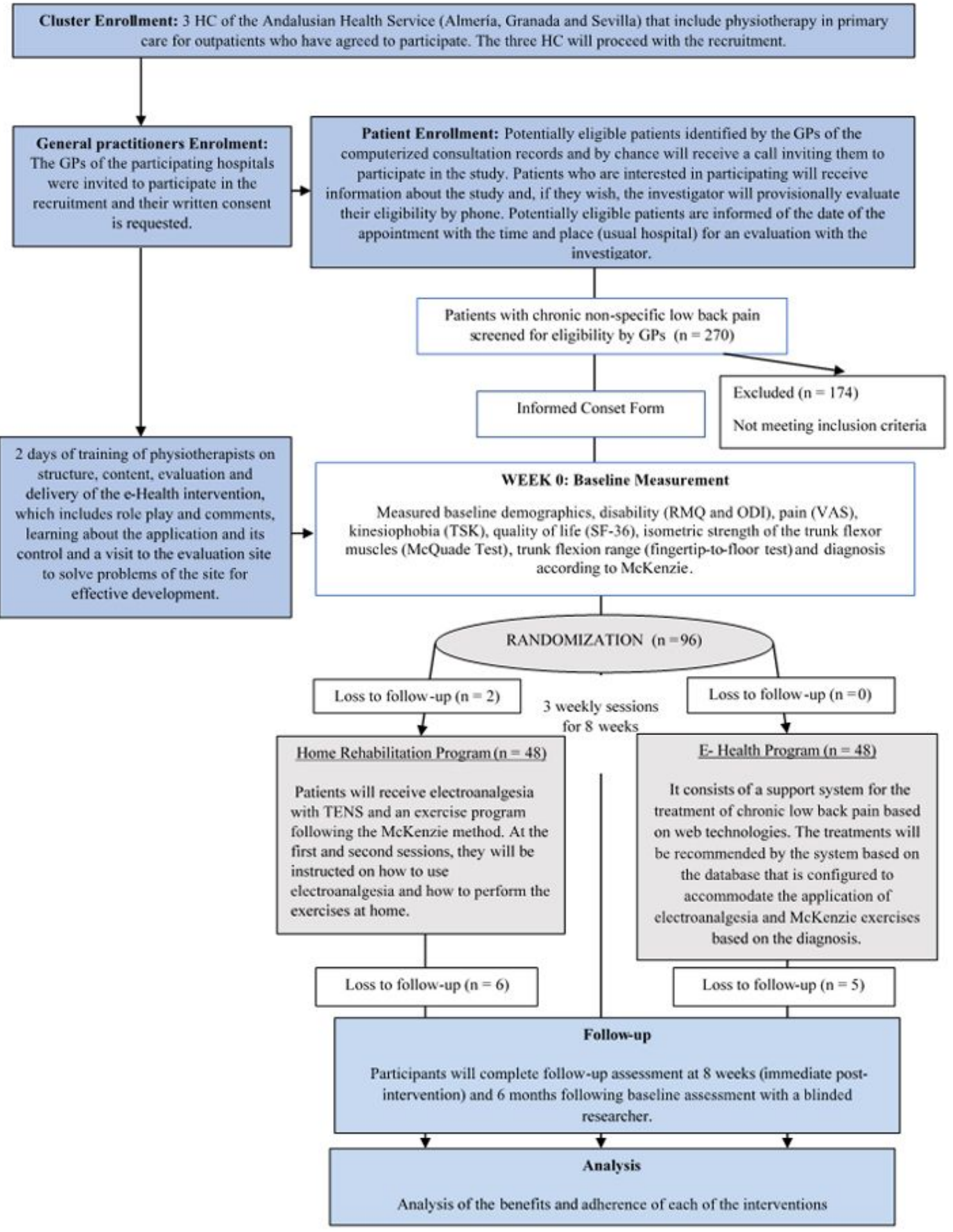


Figure 1

Design and flow of participants through the trial. *Abbreviations: RMQ = Roland-Morris Low Back and Disability Questionnaire; ODI = Oswestry Disability Index; VAS = Visual Analogue Scale; TSK = Tampa Scale for Kinesiophobia
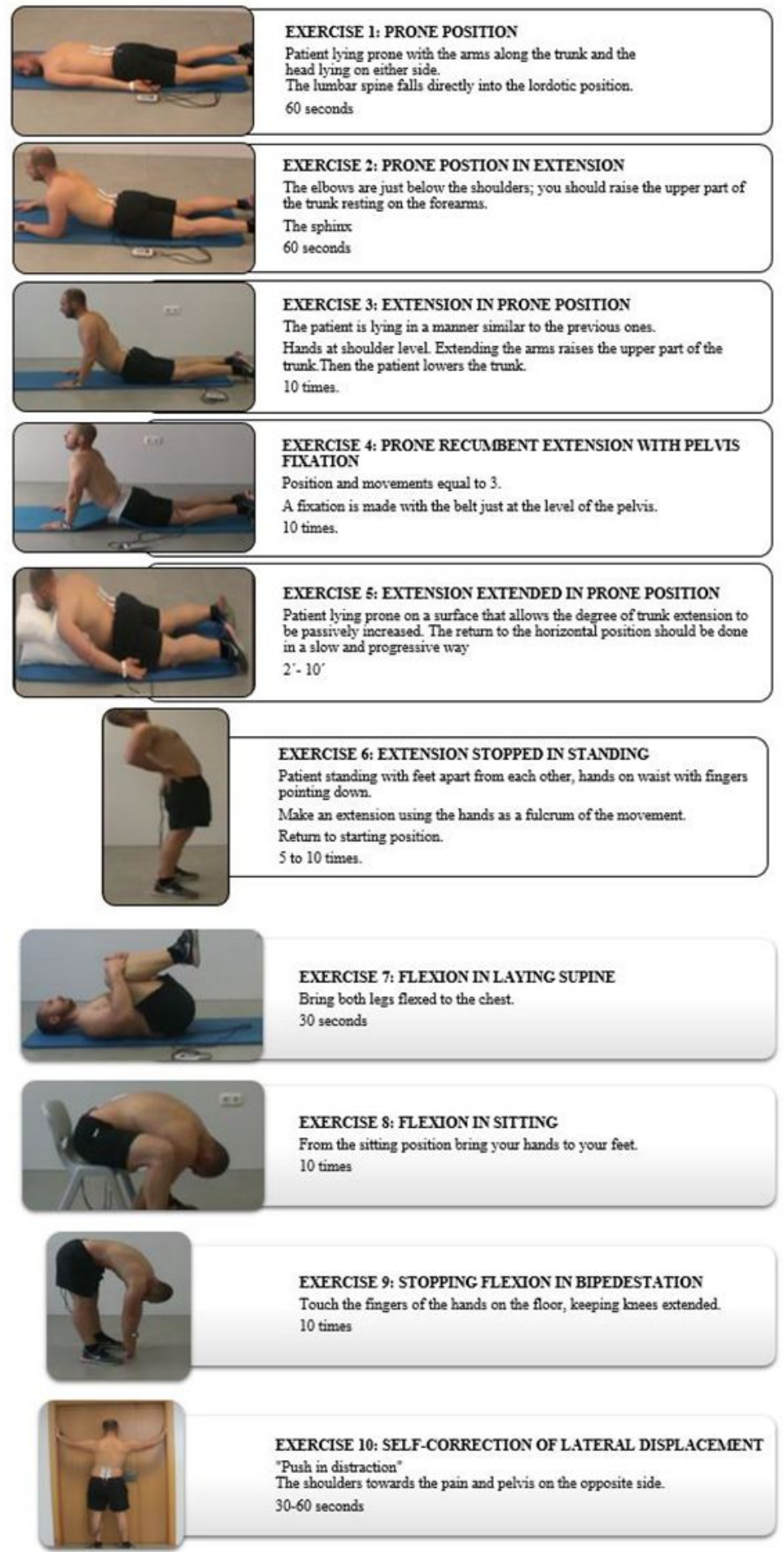

Figure 2 
Description of the McKenzie exercises prescribed for home.

1
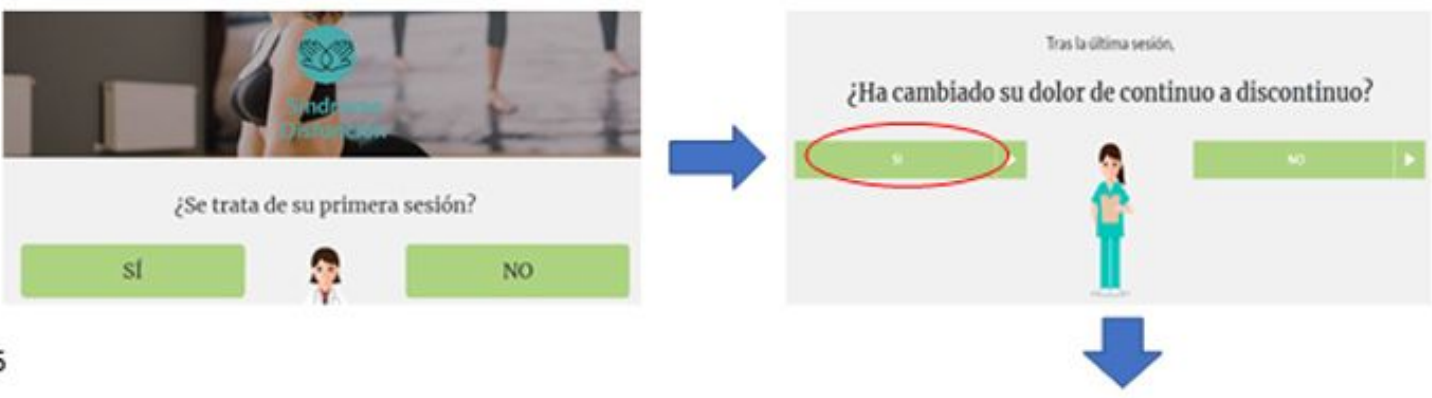

j
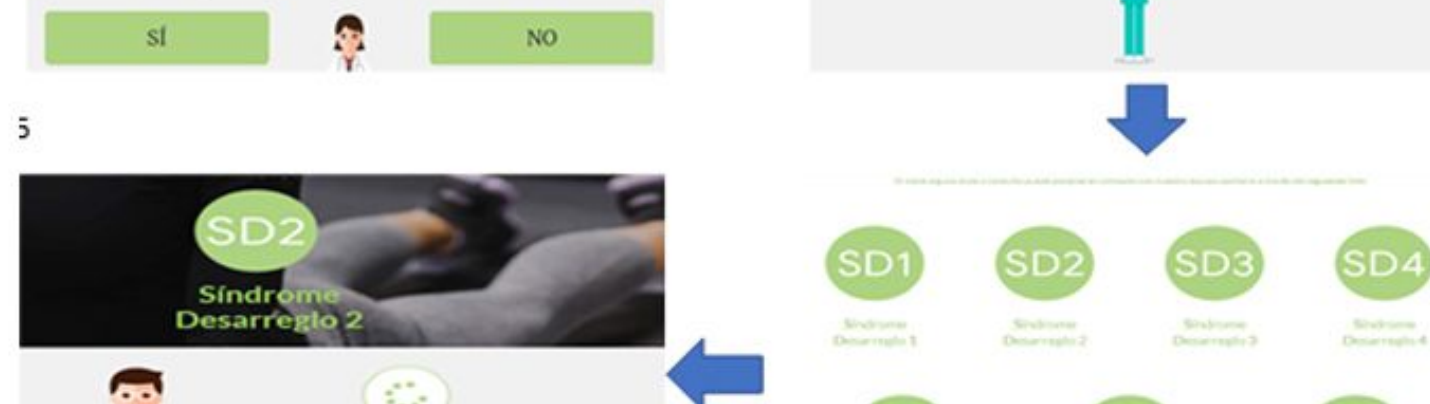

ค

son

SD5

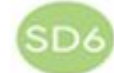

SD7

(1.

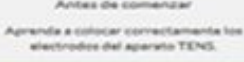

sivise.

somest.

11

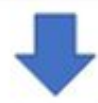

Acontinuacion

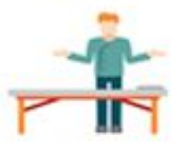

Sea ta secuenda de fier ridos propuesta tal y como se detala en los slavientes videos explcathos.

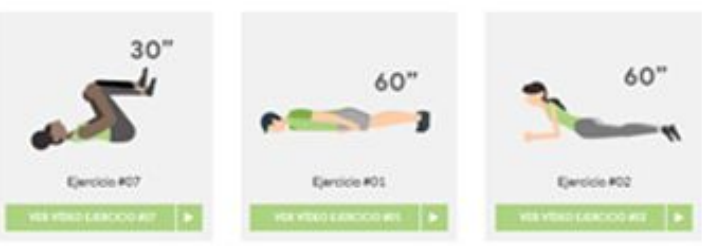

$>>>>>>>>>>>>>>>>>>>>>$
$x_{x 10}$
$3 \times 10$
$1^{x 5-10}$
$c>$
consevens
condeserer
conesense
intoses.
Envicenor

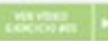

\section{Figure 3}

Treatment plan according to the symptomatic evolution of pain.

\section{Supplementary Files}


This is a list of supplementary files associated with this preprint. Click to download.

- SPIRITchecklist.docx 